\title{
Clinical challenges in interpreting multiple pathogenic mutations in single patients
}

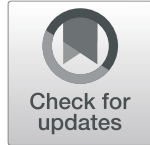

Christa Slaught ${ }^{1}$, Elizabeth G. Berry ${ }^{1 *}$ D, Lindsay Bacik ${ }^{2}$, Alison H. Skalet ${ }^{1,3,4,5}$, George Anadiotis ${ }^{6}$, Therese Tuohy ${ }^{6}$ and Sancy A. Leachman ${ }^{1,4}$

\begin{abstract}
Background: In the past two decades, genetic testing for cancer risk assessment has entered mainstream clinical practice due to the availability of low-cost panels of multiple cancer-associated genes. However, the clinical value of multiple-gene panels for cancer susceptibility is not well established, especially in cases where panel testing identifies more than one pathogenic variant. The risk for specific malignancies as a result of a mutated gene is complex and likely influenced by superimposed modifier variants and/or environmental effects. Recent data suggests that the combination of multiple pathogenic variants may be fewer than reported by chance, suggesting that some mutation combinations may be detrimental. Management of patients with "incidentally" discovered mutations can be particularly challenging, especially when established guidelines call for radical procedures (e.g. total gastrectomy in $\mathrm{CDH1}$ ) in patients and families without a classic clinical history concerning for that cancer predisposition syndrome.
\end{abstract}

Case presentation: We present two cases, one of an individual and one of a family, with multiple pathogenic mutations detected by multi-gene panel testing to highlight challenges practitioners face in counseling patients about pathogenic variants and determining preventive and therapeutic interventions.

Conclusions: Ongoing investigation is needed to improve our understanding of inherited susceptibility to disease in general and cancer predisposition syndromes, as this information has the potential to lead to the development of more precise and patient-specific counseling and surveillance strategies. The real-world adoption of new or improved technologies into clinical practice frequently requires medical decision-making in the absence of established understanding of gene-gene interactions. In the meantime, practitioners must be prepared to apply a rationale based on currently available knowledge to clinical decision-making. Current practice is evolving to rely heavily on clinical concordance with personal and family history in making specific therapeutic decisions.

Keywords: Melanoma, Genetics, Compound heterozygosity, BAP-1, MSH6, RECQL4

\section{Background}

Use of multi-gene panel testing (MGPT) with nextgeneration sequencing (NGS) for the diagnosis of hereditary cancer predisposition has increased significantly over recent years [1]. Due to the rise in utilization and affordability of MGPT, more individuals are being

\footnotetext{
*Correspondence: berryel@ohsu.edu

'Department of Dermatology, Oregon Health \& Science University, 3303 SW Bond Ave, Suite 16D, Portland, OR 97239, USA

Full list of author information is available at the end of the article
}

identified with inherited germline mutations in what are believed to be well-described, moderately and highly penetrant genes. The phenomenon may be rare. A 2018 review of $B R C A 1$ and $B R C A 2$ double heterozygotes estimated the frequency of occurrence in the nonAshkenazi Jewish population of 1:190,000 [2]. Recent evidence presented by one of the larger germline cancer predisposition clinical testing companies (Invitae) showed that $\sim 5 \%$ of 264 pancreatic cancer patients who tested positive for pathogenic or likely pathogenic 
mutations in a panel of 84 genes had 2 or more such mutations [3]. Although statistical significance cannot be assessed, the results may be fewer than reported by chance, suggesting that some genetic combinations of mutations may be detrimental.

Often, these patients do not meet established clinical criteria for a known cancer syndrome. Accurate prediction of the functional consequences of identified genetic variants plays a vital role in the field of genetic test reporting. However, the exact effect of genetic mutations on protein function is often not known. Furthermore, the risk for specific malignancies as a result of a mutated gene/protein is complex with the potential for superimposed modifier gene and environmental effects that often remain incompletely understood. We highlight two scenarios with combinations of more than one pathogenic (or likely pathogenic) mutation to illustrate the challenges practitioners face in incorporating genetic testing results into clinical practice (Table 1):

\section{Case presentation}

Patient 1 is a 70 -year-old Caucasian woman who was referred for genetic testing given her extensive personal history of cancer as listed in Table 1. She underwent Invitae Multi-Cancer panel genetic testing (Supplemental Table 1) that detected heterozygous mutations in BRCA1-associated protein 1 (BAP1), MutS Homolog 6 (MSH6) and ATP-dependent DNA helicase Q1 (RECQ L4) genes, all noted to be "pathogenic" or "likely pathogenic."

Patient 2 underwent genetic testing due to her known familial MSH6 mutation and family history of cancer concerning for germline BAP1 mutation. Ambry genetics site-specific testing revealed only the familial BAP1 mutation in our patient. However, her youngest sister (IV-6) tested positive for both the BAP1 and MSH6 mutations.

\section{Discussion}

\section{Defining pathogenicity}

One important consideration in clinical management is the pathogenicity of the identified gene variants. "Pathogenic" and "likely pathogenic" variants imply that the mutation warrants surveillance according to full highrisk guidelines and qualifies for predictive testing of atrisk relatives [4]. On the other end of the spectrum, "likely non-pathogenic" and "non-pathogenic" variants suggest that the genetic change is a polymorphism or normal variant and is to be treated as if no mutation was detected [4]. Between these categories is a "variant of unknown significance," where clinical management is decided, case-by-case, based on personal and family history and other risk factors, as further understanding evolves [4, 5].
The reliability of variant classification has improved in recent years with the introduction of variant classification frameworks. In response to the observation that variant classifications could differ between laboratories, the American College of Medical Genetics and Genomics-Association for Molecular Pathology (ACMG-AMP) established guidelines to create a common framework for variant classifications in 2015 [6]. In 2017, "Sherloc" (semiquantitative, hierarchical evidencebased rules for locus interpretation) was developed as a refinement of the ACMG-AMP criteria [7]. All interpreted variants are routinely deposited into ClinVar, for ease of access.

With more widespread use of MGPT, there are also increasing reports of individuals with pathogenic mutations in multiple cancer predisposition genes. A recent review of 55,803 patients tested with a 25-hereditary cancer gene panel, found that $106(0.19 \%)$ had pathogenic or likely pathogenic mutations in two or more genes [8].

Our patients' mutations were deemed likely pathogenic or pathogenic (Table 1). MSH6, BAP1, and RECQ L4 mutations are rare individually. To our knowledge, the combinations of genes inherited in these individuals has never been reported.

\section{Review of mutations \\ BAP1}

The BAP1 gene encodes a nuclear-localized deubiquitinase and plays a pivotal role in epigenetic modification, transcription regulation, and DNA damage response [9]. Germline mutations of $B A P 1$ are associated with an autosomal dominant, novel cancer syndrome characterized by atypical Spitz tumors (AST), uveal melanoma, mesothelioma (MM), clear cell renal cell carcinoma, cutaneous melanoma $(\mathrm{CM})$, and basal cell carcinoma (BCC) [9-13].

The molecular mechanisms and cellular pathways responsible for leading to these specific tumor types remain unclear [13]. The full spectrum of this syndrome is still being characterized and more recently associated tumors include meningioma, cholangiocarcinoma, hepatocellular carcinoma, lung cancer, and breast/ovarian cancer [13, 14]. Data remains limited regarding accurate estimations of either the lifetime risks or average age of diagnosis for each of these associated cancers $[12,15,16]$.

As the full phenotype for mutations in this gene needs additional investigation, numerous screening guidelines have been proposed (Table 2). Screening recommendations support at least annual general physical, ophthalmological and dermatologic examinations [10, 11, 15]. Given the impairment in DNA damage repair associated with BAP1 mutations, it is suggested that ultrasound 


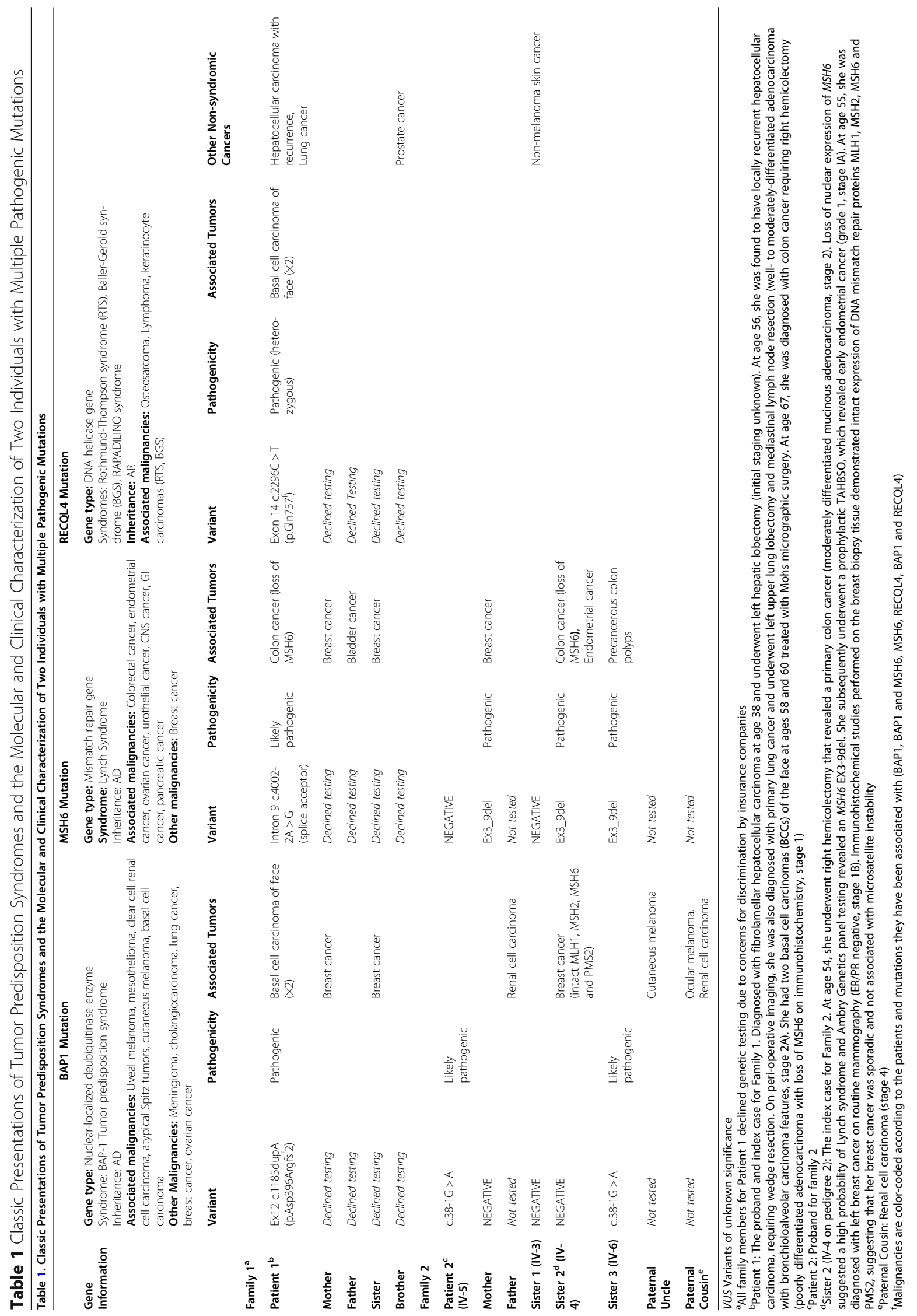


Table 2 Malignancy Screening Recommendations by Gene

\begin{tabular}{|c|c|c|c|}
\hline & Associated Neoplasms & $\mathrm{Age}^{\mathrm{a}}$ & Recommendation \\
\hline \multirow[t]{4}{*}{ BAP1 } & Uveal melanoma & 11 & Annual dilated eye exams by an ocular specialist \\
\hline & Mesothelioma & - & Annual physical +/- Chest MRI \\
\hline & Cutaneous melanoma, BCC, AST & 20 & Annual or biannual full body skin exam \\
\hline & Clear cell renal cell carcinoma & - & $\begin{array}{l}\text { Annual physical } \\
\text { Annual abdominal ultrasound } \\
\text { Annual urinalysis } \\
\text { Abdominal MRI every } 2 \text { years }\end{array}$ \\
\hline \multirow[t]{7}{*}{ MSH6 } & Colon cancer & 25 & Colonoscopy every $1-2$ years \\
\hline & Endometrial cancer & & Prophylactic hysterectomy \\
\hline & Ovarian cancer & 30 & $\begin{array}{l}\text { Prophylactic bilateral salpingo-oophorectomy } \\
\text { Prior to prophylactic removal, consider: } \\
\text { Annual transvaginal ultrasound } \\
\text { Annual CA-125 }\end{array}$ \\
\hline & Urothelial cancer & 30 & Consider annual urinalysis \\
\hline & Gastrointestinal tract & 30 & Esophagogastroduodenoscopy every $3-5$ years \\
\hline & Pancreatic cancer & - & Consider endoscopic ultrasound \\
\hline & CNS malignancy & & Annual physical exam with neurologic examination \\
\hline \multirow[t]{3}{*}{$\begin{array}{l}\text { RECQL4 (biallelic) } \\
\text { (RTS, BGS, RAPADILINO } \\
\text { Syndrome) }\end{array}$} & Osteosarcoma & & $\begin{array}{l}\text { Annual physical } \\
\text { Prompt skeletal radiographic evaluation when suspected } \\
\text { clinically }\end{array}$ \\
\hline & Lymphoma & & $\begin{array}{l}\text { Annual physical } \\
\text { Baseline complete blood count with differential }\end{array}$ \\
\hline & $\begin{array}{l}\text { Keratinocyte carcinomas (RTS and BGS } \\
\text { only) }\end{array}$ & & Annual full body skin exam \\
\hline
\end{tabular}

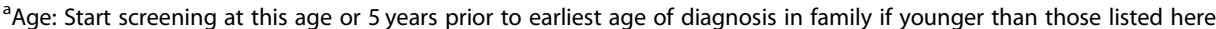
RTS Rothmund-Thompson Syndrome

$B G S$ Baller-Gerold Syndrome

and MRI be utilized, when possible, to avoid the radiation associated with CT scans [16]. Other important prevention strategies include photoprotection to minimize ultraviolet radiation exposure ( $\mathrm{CM} / \mathrm{BCC}$ risk), avoidance of arc welding (UM risk) and avoidance of smoking or asbestos exposure (MM risk) [16].

\section{MSH6}

The MSH6 gene encodes one protein member of a growing number of heteroduplex complexes of proteins that maintain genomic stability by recognizing and correcting single base mismatches and insertion/deletion loops that may arise during replication [4]. Heterozygous germline mutations in any one of several mismatch repair genes are associated with Lynch syndrome, an autosomal dominant cancer-susceptibility disorder previously known as hereditary nonpolyposis colorectal cancer syndrome. Approximately $90 \%$ of mutations associated with Lynch syndrome are located in $M L H 1, M S H 2$ and EPCA $M$ genes, while $10 \%$ are located in MSH6 and PMS2 (and a very small number in the more recently identified MSH3) [17]. Carriers of these mutations are at high risk of early-onset colorectal and endometrial cancer as well as tumors of the ovaries, urothelium, CNS and entire gastrointestinal tract, including the pancreas [17].
Multiple national and international professional screening recommendations have been proposed (Table 2) [18]. Although data indicate a mildly increased risk for breast cancer, no specific recommendations have been made for this risk.

\section{RECQL4}

RECQL4 is a DNA helicase gene that has been implicated in DNA double stranded break repair, nucleotide excision repair, and base excision repair, important for DNA replication and repair of UV damage [19-22]. This gene may also play a role in telomere maintenance [23]. Homozygous (or compound heterozygous) germline mutations in the RECQL4 gene have been associated with Rothmund-Thompson (RTS), Baller-Gerold (BGS), and RAPADILINO syndromes [24]. These syndromes are autosomal recessive conditions associated with biallelic disruption of the helicase gene (usually compound heterozygous or consanguineous homozygous mutations). RTS, BGS, and RAPADILINO have been associated (albeit rarely) with osteosarcoma and lymphoma, and both RTS and BGS are associated with skin cancer, specifically keratinocyte carcinomas [25]. Thus, in the case of biallelic loss of gene function, evidence suggests an association of RECQL4 with cancer predisposition [23]. The 
impact of a heterozygous RECQL4 mutation on cancer predisposition unknown, particularly in the context of simultaneously inherited DNA damage and repair gene mutations like BAP1 and/or MSH6.

Table 2 includes a summary of the screening recommendations for malignancies associated with RECQL4. In addition to regular screening, photoprotection and minimization of ultraviolet radiation may be important. We recommend a conservative approach to UV and other types of radiation, as the inevitable somatic loss of RECQL4 in random heterozygous cells may lead to increased risks for clonal populations of those cells over time. This idea is supported by a recent report that heterozygous germline carriers of pathogenic RECQL4 mutations were over-represented in children with osteosarcoma [26].

\section{Multiple pathogenic mutations}

Compared to phenotype-driven single gene testing, MGPT increases the probability of identifying pathogenic mutations. Studies of large populations of women diagnosed with breast cancer suggest that nearly $8 \%$ carry a pathogenic variant that, if detected, would warrant a change in preventive care, such as secondary breast cancer screening incorporating MRI, early colonoscopy or high risk surgery [27]. In addition, as many as $4 \%$ of these individuals have germline pathogenic mutations in cancer predisposition genes other than BRCA1 and BRCA2 on MGPT (i.e. CHEK2, PALB2, ATM, NBN, PTEN, etc.) $[27,28]$. Nevertheless, the benefit of more comprehensive genetic testing strategies is often debated. Concerns include cost, increased procedurerelated morbidity, and the potential for discovery of uninformative or anxiety-provoking results [29].

Another potential consequence of MGPT is the identification of more than one pathogenic mutation in the same individual or identification of pathogenic mutations that do not match the patient's phenotype. Review of larger cohorts undergoing MGPT for breast, ovarian and general hereditary cancer risk has revealed that approximately $3 \%$ of individuals test positive for pathogenic mutations in multiple genes [30]. This carries significance for both the individual and their family members, suggesting that single-gene testing-even if targeted to known familial pathogenic mutations-may miss other significant pathogenic mutations. A review looking specifically at patients undergoing genetic testing for suspected Lynch syndrome based on history of Lynch syndrome-associated cancer and/or polyps, found that MGPT identified high-penetrance mutations in other non-Lynch Syndrome cancer predisposition genes in $5.6 \%$ of these individuals, many of which were unexpected based on patients' histories [31]. In fact, that study found more than one high-penetrance non-Lynch
Syndrome gene mutation for every 5 Lynch Syndrome gene mutations identified, revealing that it is not uncommon to have unexpected, clinically useful findings with MGPT in this population [31]. MGPT should be considered to identify missed pathogenic mutations and more accurately inform hereditary cancer risk.

Identifying patients with multiple clinically actionable mutations has important implications for patients and their family members, yet much remains unclear regarding the consequence of carrying more than one pathogenic mutation. Our understanding of how combinations of mutations may interact to alter the ultimate profile of cancers in a patient, or their family, is currently very limited [32-34]. Certain combinations of mutations may be detrimental in that they increase overall risk of malignancy while others may reduce overall cancer risk, relative to inheritance of the single gene mutation alone. In addition, the remainder of any individual's genomic background and their cumulative exposure history to cancer-predisposing agents superimpose additional effects on the risks conferred by the high-penetrance genes.

Based on this framework and the limited data we have available; a few effects are possible:

1) Detrimental consequence: Some germline cancer susceptibility mutations may interact in an additive or synergistic manner to increase the penetrance of cancers related to other germline cancer susceptibility mutations. There is some precedence for this in the literature. The rs2304277 variant in the OGG1 glycosidase gene of the base excision repair pathway has been shown to increase the penetrance of ovarian cancer in patients with $B R C A 1 / 2$ mutations [35]. The mutation was found to independently decrease expression of mRNA OGG1, thus contributing to further genomic instability via DNA damage and telomere shortening [35]. In addition, co-inheritance of the cyclin-dependent kinase inhibitor (CDKN2A) gene and variants of the melanocortin-1 receptor (MC1R) gene that have previously been associated with red hair, fair skin and sensitivity to ultraviolet light (Arg151Cys, Arg160Trp, and Asp294His) have been found to significantly increase melanoma penetrance and decreased the age of onset by 20 years compared to individuals carrying the CDKN2A mutation alone [36]. Earlier onset of colorectal cancer has also been reported in an individual with both compound heterozygous MSH6 mutations and an APC missense mutation, diagnosed at age 18 [37].

2) Beneficial consequence: Increased fragility of cells carrying multiple mutations could counterintuitively result in an overall cancer risk 
that is less than the sum of the individual risks from the inherited germline mutations. There may be a restricted profile of tertiary and subsequent mutations that a cell can handle. This may lead to increased apoptosis, cell death or senescence as somatic mutations accumulate, which could reduce penetrance or improve prognosis of tumors. For example, as outlined above, germline mutations affecting DNA repair genes, such as MSH6, have been clearly associated with a high risk of cancer development [17]. However, gene expression profiling of tumor cell lines and specimens of different histologic origin has also shown that overexpression of DNA repair genes is often associated with more aggressive behavior of cancer cells and lower patient disease-free or overall survival, including melanoma [38]. These findings have led to the hypothesis that, while genetic instability is essential for tumor initiation, it may decrease the potential for progression or metastasis [39].

3) Genomic Background and Environmental Exposure: In addition to the consequence that inherited germline mutations may have on each other, the overall penetrance of a cancer predisposition syndrome is also affected by the individuals genomic background and environmental exposures, such as ultraviolet radiation. For example, protective modifier genes may exist that mitigate the risks conferred by high-penetrance genes. A recent analysis of 589,306 genomes by Friend et al. identified 8 individuals resilient to severe Mendelian childhood diseases [40]. These individuals had known pathogenic mutations for highly penetrant diseases, yet had not reported clinical manifestations of the disease. They postulate that protective genetic variants may be identifiable that are acting to attenuate the expression of disease in these individuals.

Various combinations of germline cancer susceptibility mutations most likely interact in unique ways. Some combinations may potentiate risk for specific malignancies while others may attenuate risk, perhaps depending on specific pathway interactions. This is likely to create difficulty in ascribing a particular cancer syndrome to a family or individual. As genetic testing becomes more widely available and used, it will be critical to expand our knowledge of the interactions among germline mutations and the ultimate genotype-phenotype consequences.

As costs of MGPT decrease, the risk of missing pathogenic mutations may outweigh the arguments against panel testing. MGPT offers lower cost of testing per gene [41]. However, the summative costs of genetic testing should take in to account the cost of screening and heightened surveillance for each positive result. On a societal level, the added costs of screening will need to be evaluated in the context of earlier diagnosis and treatment of the associated malignancies. For the individual, while insurance generally covers the costs of National Comprehensive Cancer Network (NCCN) guidelinesupported risk management for known pathogenic mutations, costs may be of particular concern to uninsured or underinsured patients. Furthermore, discussion of patient concerns regarding genetic privacy and federal and state laws is needed.

While our understanding of cancer heritability, and particularly the interplay among multiple pathogenic mutations, remains unclear, it is important to acknowledge and accept the uncertainty while striving to advance the field toward a more comprehensive understanding. Prior to initiating testing, it is important to ensure that both the counselor and the patient are comfortable with results of unknown significance [16]. Ideally, physicians and counselors collaborate with patients throughout the process to explain fully the known and yet unknown before working with them to make informed, individualized plans for ongoing care and surveillance based on the profile of cancers that have been reported in association with the mutations. With time, ongoing clarifications of genotype-phenotypeenvironment relationships will result in adjustments to current clinical screening guidelines and allow for more accurate personalized surveillance.

\section{Screening our patients}

While our understanding of the interplay among combinations of germline cancer susceptibility gene mutations remains incomplete, we need to be prepared to provide counseling and screening recommendations to the best of our abilities. Available screening guidelines for each relevant mutation should be reviewed (Table 2), however, it is critical that we individualize care to each patient. A review of consensus guidelines, emerging guidelines and a patient's family history of cancer should all be considered when working with patients to make informed and shared decisions on screening.

Management of patients with "incidentally" discovered mutations can be particularly challenging, especially when established guidelines call for radical procedures (e.g. total gastrectomy in CDH1) in patients and families without a classic clinical history concerning for that cancer predisposition syndrome. For this reason, current practice is evolving to rely on clinical concordance with personal and family history in making specific screening or therapeutic decisions. In the case that genetic test results reveal a pathogenic or likely pathogenic mutation in an individual without a personal or family history 
suggestive of that particular cancer pre-disposition syndrome, less rigid screening protocols could be considered. For example, if a patient tests positive for a pathogenic mutation in MSH6 but does not have a personal or family history suggestive of classic Lynch Syndrome, delay of initial colonoscopy to age $25-30$ or less frequent colonoscopies could be considered. Cascade testing may also be discouraged in extended family.

In the case that an individual has a family history of cancers that lie outside of the established spectrum of cancer predisposition identified by germline mutations, the 10-year rule may be utilized. Screening for that particular malignancy may be instituted when the individual is 10 years younger than the earliest known diagnosis in the family. This 10-year rule may be applied to first- and second-degree relatives.

\section{Patient 1}

MGPT was performed after her colon cancer had negative IHC staining for MSH6, and confirmed a germline mutation in $\mathrm{MSH6}$, while revealing additional mutations in BAP1 and RECQL4 genes (Table 1, Fig. 1). Although she had an extensive personal history of cancer suggestive of cancer predisposition, her clinical phenotype and family history would not have predicted either $B A P 1$ or RECQL4 mutations prior to testing.

Her screening regimen consists of a combination of the recommendations for BAP1 Tumor Predisposition Syndrome and Lynch Syndrome, respectively. She is seen by dermatology every 6 months for full body skin examination (risk for melanoma and basal cell skin cancer with $B A P 1$ ), ophthalmology every 6 months for dilated fundus examination and annually for ultrasound biomicroscopy (UBM) (risk for uveal melanoma with BAP1). She also undergoes annual renal ultrasound, and MRI chest and abdomen every 2 years (risk for internal malignancies associated with $B A P 1$, including mesothelioma and renal cell carcinoma) [13]. She continues annual colonoscopy and mammogram (risk for colon and breast cancer in Lynch Syndrome). She had a hysterectomy prior to her diagnosis of Lynch syndrome, but pelvic ultrasound and CA-125 are monitored annually (risk for

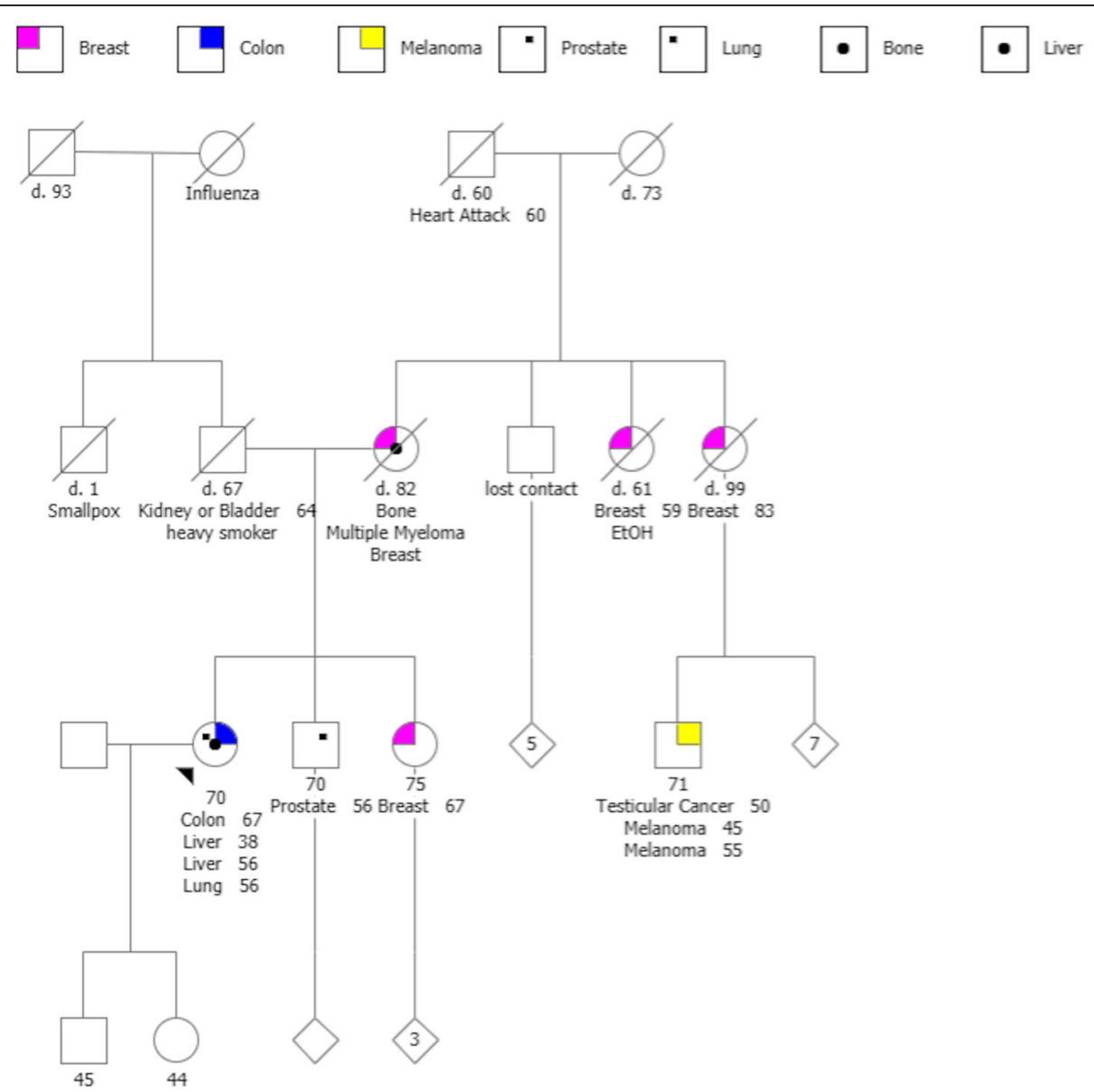

Fig. 1 Pedigree for Family 1. The arrow indicates Patient 1, the proband and index case for Family 1. Her genetic testing revealed: BAP 1: Exon 12 c.1185dupA (p.Asp396Argfs*2), pathogenic MSH6: Intron 9 c.4002-2A > G (splice acceptor), likely pathogenic. RECQL4: Exon 14 c.2296C > T $\left(\mathrm{p} . \mathrm{G} \ln 757^{*}\right)$, pathogenic (heterozygous). All other family members declined genetic testing 
ovarian carcinoma). UV-minimization has been emphasized given her history of both BAP1 and RECQL4 mutations. No specific screening for osteosarcoma is performed.

\section{Patient 2}

The sisters in Family 2 demonstrate a Mendelian inheritance pattern of the two familial cancer predisposition mutations in MSH6 and BAP1 (Table 1, Fig. 2). One sister (IV-3) did not inherit either mutation; one (IV-4) inherited just the MSH6 mutation, one (IV-5, the proband in this study) inherited just the BAP1 mutation, and one (IV-6) inherited both MSH6 and BAP1 mutations.

The arrow indicates Patient 2, the proband for Family 1. The sisters in generation IV of Family 2 demonstrate a Mendelian inheritance pattern of the two familial cancer predisposition mutations in MSH6 and BAP1 (MSH6: Ex3_9del, pathogenic; BAP1: c.38-1G > A, likely pathogenic). One sister (IV-3) did not inherit either mutation; one (IV-4) inherited just the MSH6 mutation (index case for Family 1), one (IV-5, the proband in this study) inherited just the BAP1 mutation, and one (IV-6) inherited both MSH6 and BAP1 mutations.

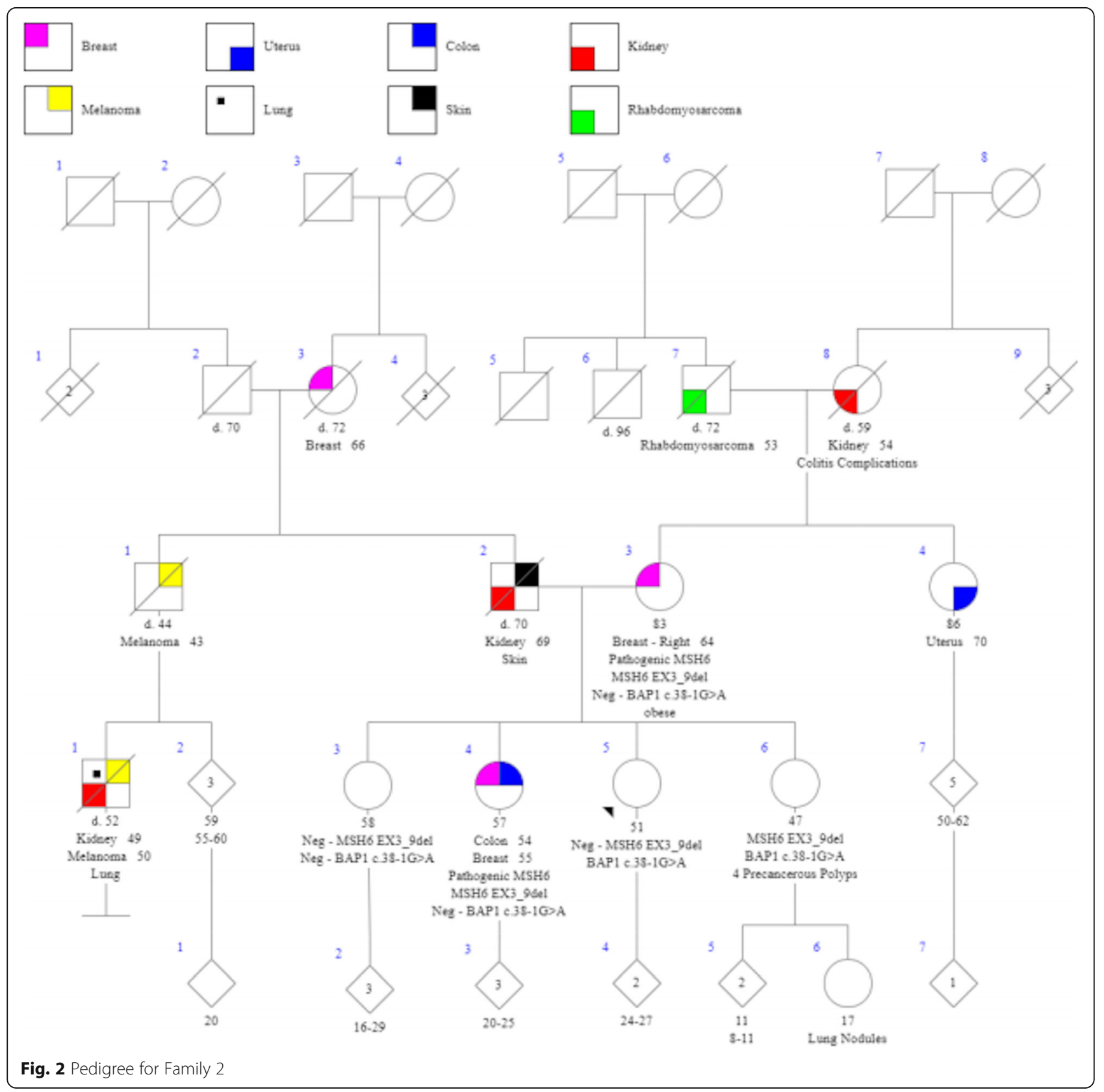


Our patient was found to have a BAP1 c.38-1G > A likely pathogenic variant and site-specific testing for the familial MSH6 mutation was negative. She is screened with full body skin examinations every 6 months, dilated fundus examinations every 6 months with annual UBM, annual renal ultrasound and MRI chest and abdomen every 2 years.

The index case (IV-4) had a history of a tubular adenoma in the hepatic flexure, several small adenomas, a hyperplastic polyp, and two tubular adenomas in the ascending colon and sigmoid colon at age 51; then a tubular adenoma in the sigmoid colon and a right hepatic mass with no definitive invasive carcinoma at 54 . She underwent a prophylactic right hemicolectomy a month later that revealed a $2.6-\mathrm{cm}$ moderately differentiated mucinous adenocarcinoma, with invasion through the muscularis propria and superficially into subserosa adipose tissue (Stage 2). Loss of nuclear expression of MSH6 suggested a high probability of Lynch syndrome, in the absence of a previous family history of colorectal cancer. Ambry Genetics panel testing revealed an $\mathrm{MSH} 6$ EX3-9del. She subsequently underwent a prophylactic total abdominal hysterectomy with bilateral salpingooophorectomy (TAHBSO), which revealed a Stage IA, grade 1 endometrial cancer at 54. At 55, she was diagnosed with a Stage 1B ER/PR-negative left-sided breast cancer, following routine mammography. Immunohistochemical studies performed on the breast biopsy tissue demonstrated intact expression of DNA mismatch repair proteins MLH1, MSH2, MSH6 and PMS2, suggesting that her breast cancer was sporadic and not associated with microsatellite instability.

The youngest sister (IV-6) was found to have both familial mutations after testing for a broad profile (Supplemental Table 2). She was noted to have a personal history of 4 precancerous colorectal polyps at the time of her genetics visit. She has decided to undergo a combination of both screening regimens but has yet to manifest a malignancy at age 47.

The eldest sister (IV-3) tested negative for both familial mutations through site-specific testing for the familial variants through Ambry Genetics.

\section{Conclusions}

Cancer genetics is advancing quickly as a tool for precision medicine as we move into an era of whole-exome and whole-genome sequencing. With large panel testing becoming more widely available and affordable, the challenge of counseling and managing patients with mutations in multiple germline cancer susceptibility genes, with incompletely understood interactions, will become more prevalent. To address our questions and uncertainties, we must be prepared to track, research and share our insights into the human genome. As we work to advance the field toward a more comprehensive understanding of predictive cancer genetics and the complex interplay of other genomic and environmental exposure effects, it is important that we work to individualize care for each patient while recommending a rational plan based on existing data.

We support broad MGPT in assessing patients and families for inherited predisposition to cancer and other diseases. The practice of clinical genetics is in a period of rapidly advancing understanding of genotypephenotype correlations. While this raises puzzling and challenging questions, only through more detailed, open-ended testing and meticulous data collection will we better tease apart the complex results. Ironically, this conundrum highlights the continued relevance and importance of family history as a practical guide, despite the immense and improving technology at our disposal.

\section{Supplementary Information}

The online version contains supplementary material available at https://doi. org/10.1186/s13053-021-00172-3.

Additional file 1. Invitae Multi-Cancer Panel. Broad Profile Testing for Patient 2's youngest sister (IV-6).

\section{Abbreviations \\ MGPT: Multi-gene panel testing; NGS: Next-generation sequencing; \\ VUS: Variants of unknown significance; BAP-1: BRCA-1 associated protein 1; MSH6: MutS Homolog 6; ACMG-AMP: American College of Medical Genetics and Genomics-Association for Molecular Pathology; AST: Atypical Spitz tumors; MM: Malignant mesothelioma; CM: Cutaneous melanoma; BCC: Basal cell carcinoma; RTS: Rothmund-Thompson Syndrome; BGS: Baller-Gerold Syndrome; CDKN2A: Cyclin-dependent kinase inhibitor; MC1R: Melanocortin- 1 receptor; MRI: Magnetic resonance imaging; IHC: Immunohistochemistry; NCNN: National Comprehensive Cancer Network; UBM: Ultrasound biomicroscopy; TAHBSO: Total abdominal hysterectomy with bilateral salpingo-oophorectomy}

\section{Acknowledgements}

John D. Gray, MD Endowed Chair in Melanoma Research through Knight Cancer Institute to support efforts of Sancy Leachman, MD PhD

\section{Authors' contributions}

CS, EGB, LB and TT collected and analyzed the data and were major contributors to writing the manuscript with oversight and direction by SAL. AHS, GA and SAL contributed significantly to revisions of the manuscript. All authors read and approved the final manuscript.

\section{Funding}

SAL is supported by grant P30 CA069533-21 from the National Institutes of Health, Title: OHSU Knight Cancer Institute, PI: Druker, Brian J. AHS is supported by grant P30 EY010572 from the National Institutes of Health (Bethesda, MD), and by unrestricted departmental funding from Research to Prevent Blindness (New York, NY). These funding sources had no role in the study and collection, analysis or interpretation of data, or writing of manuscript.

\section{Availability of data and materials}

The datasets used and/or analyzed during the current study are available from the corresponding author on reasonable request.

Ethics approval and consent to participate

The need for IRB review was waived by the IRB at Oregon Health and Science University (STUDY00018664). 


\section{Consent for publication}

Obtained from the individuals reported.

\section{Competing interests}

The authors declare that they have no competing interests.

\section{Author details}

'Department of Dermatology, Oregon Health \& Science University, 3303 SW Bond Ave, Suite 16D, Portland, OR 97239, USA. ${ }^{2}$ Department of Dermatology, Penn State Health, Hershey, USA. ${ }^{3}$ Department of Ophthalmology, Casey Eye Institute, Oregon Health \& Science University, Portland, USA. ${ }^{4}$ Knight Cancer Institute, Oregon Health \& Science University, Portland, USA. ${ }^{5}$ Department of Radiation Medicine, Oregon Health \& Science University, Portland, USA. ${ }^{6}$ Legacy Cancer Institute, Cancer Genetics Services, Legacy Health Systems, Portland, USA

Received: 12 June 2020 Accepted: 25 January 2021

Published online: 04 February 2021

\section{References}

1. Espenschied CR, LaDuca H, Li S, McFarland R, Gau C-L, Hampel H. Multigene panel testing provides a new perspective on lynch syndrome. J Clin Oncol. 2017;35(22):2568-75

2. Palmirotta R, Lovero D, Stucci LS, Silvestris E, Quaresmini D, Cardascia A, Silvestris F. Double heterozygosity for BRCA1 pathogenic variant and BRCA2 polymorphic stop codon K3326X: a case report in a southern Italian family. Int J Mol Sci. 2018;19(1):285.

3. Esplin, E. Increasing Access for Patients With Pancreatic Cancer to Germline Genetic Testing: Clinical Impact Across Disease Stage and Ethnicity. Oral presenation at: 39th Annual National Society of Genetic Counselors Meeting; November 2020; Virtual.

4. Peltomäki P. Update on lynch syndrome. Familial Cancer. 2016;15(3):385-93.

5. Thompson BA, Spurdle AB, Plazzer J-P, et al. Application of a five-tiered scheme for standardized classification of 2,360 unique mismatch repair gene variants lodged on the InSiGHT locus-specific database. Nat Genet. 2014;46(2):107-15.

6. Maxwell KN, Hart SN, Vijai J, et al. Evaluation of ACMG-guideline-based variant classification of Cancer susceptibility and non-Cancer-associated genes in families affected by breast Cancer. Am J Hum Genet. 2016;98(5): $801-17$

7. Nykamp K, Anderson M, Powers M, et al. Sherloc: a comprehensive refinement of the ACMG-AMP variant classification criteria. Genet Med. 2017;19(10):1105-17.

8. Weitzel JN, Blazer KR, Nehoray B, et al. Assessment of the clinical presentations of patients with at least two deleterious mutations on multigene panel testing. J Clin Oncol. 2015;33(15):1514.

9. Wang XY, Wang Z, Huang JB, et al. Tissue-specific significance of BAP1 gene mutation in prognostic prediction and molecular taxonomy among different types of cancer. Tumour Biol. 2017;39(6):1010428317699111.

10. Ransohoff KJ, Jaju PD, Tang JY, Carbone M, Leachman S, Sarin K. Familial skin cancer syndromes: increased melanoma risk. J Am Acad Dermatol. 2016;74(3):423-34.

11. Rojek NW, Korcheva V, Leachman SA. A large skin-colored nodule on the plantar foot: a quiz. Acta Derm Venerol. 2017;97(10):1265-6.

12. Carbone M, Ferris LK, Baumann F, et al. BAP1 cancer syndrome: malignant mesothelioma, uveal and cutaneous melanoma, and MBAITs. J Transl Med. 2012;10:179

13. Masoomian B, Shields JA, Shields CL. Overview of BAP1 cancer predisposition syndrome and the relationship to uveal melanoma. J Curr Ophthalmol. 2018;30(2):102-9.

14. Chau C, van Doorn R, van Poppelen NM, van der Stoep N, Mensenkamp AR, Sijmons RH, van Paassen BW, van den Ouweland AMW, Naus NC, van der Hout AH, Potjer TP, Bleeker FE, Wevers MR, van Hest LP, MCJ J, Marinkovic M, Bleeker JC, Jager MJ, GPM L, Nielsen M. Families with BAP1-Tumor Predisposition Syndrome in The Netherlands: Path to Identification and a Proposal for Genetic Screening Guidelines. Cancers (Basel). 2019;11(8):1114. https://doi.org/10.3390/cancers1 1081114 PMID: 31382694; PMCID: PMC6721807.

15. Pilarski R, Cebulla CM, Massengill JB, et al. Expanding the clinical phenotype of hereditary BAP1 cancer predisposition syndrome, reporting three new cases. Genes Chromosomes Cancer. 2014;53(2):177-82.
16. Leachman SA, Lucero OM, Sampson JE, et al. Identification, genetic testing, and management of hereditary melanoma. Cancer Metastasis Rev. 2017: 36(1):77-90

17. Bonadona V, Bonaïti B, Olschwang S, et al. Cancer risks associated with Germline mutations in genes in lynch syndrome. JAMA. 2011;305(22): 2304-10.

18. Kastrinos F, Stoffel EM. History, genetics, and strategies for cancer prevention in lynch syndrome. Clin Gastroenterol Hepatol. 2014;12(5): $715-27$.

19. Kitao S, Ohsugi I, Ichikawa K, Goto M, Furuichi Y, Shimamoto A. Cloning of two new human helicase genes of the RecQ family: biological significance of multiple species in higher eukaryotes. Genomics. 1998;54(3):443-52.

20. Kitao S, Shimamoto A, Goto M, et al. Mutations in RECQL4 cause a subset of cases of Rothmund-Thomson syndrome. Nature Genet. 1999;22(1):82-4.

21. Schurman SH, Hedayati M, Wang Z, et al. Direct and indirect roles of RECQ L4 in modulating base excision repair capacity. Hum Molec Genet. 2009; 18(18):3470-83.

22. Yin J, Kwon YT, Varshavsky A, Wang W. RECQL4, mutated in the Rothmund-Thomson and RAPADILINO syndromes, interacts with ubiquitin ligases UBR1 and UBR2 of the N-end rule pathway. Hum Molec Genet. 2004;13(20):2421-30.

23. Croteau DL, Singh DK, Ferrarelli LH, Lu H, Bohr VA. RECQL4 in genomic instability and aging. Trends Genet. 2012;28(12):624-31.

24. Siitonen HA, Sotkasiira J, Biervliet M, et al. The mutation spectrum in RECQ L4 diseases. J Hum Genet. 2009;17(2):151-8.

25. Stinco G, Governatori G, Mattighello P, Patrone P. Multiple cutaneous neoplasms in a patient with Rothmund-Thomson syndrome: case report and published work review. J Dermatol. 2008;35(3):154-61.

26. Maciaszek JL, Oak N, Chen W, Hamilton KV, RB MG, Nuccio R, Mostafavi R, Hines-Dowell S, Harrison L, Taylor L, Gerhardt EL, Ouma A, Edmonson MN, Patel A, Nakitandwe J, Pappo AS, Azzato EM, Shurtleff SA, Ellison DW, Downing JR, Hudson MM, Robison LL, Santana V, Newman S, Zhang J, Wang Z, Wu G, Nichols KE, Kesserwan CA. Enrichment of heterozygous germline RECQL4 loss-of-function variants in pediatric osteosarcoma. Cold Spring Harb Mol Case Stud. 2019;5(5):a004218. https://doi.org/10.1101/mcs. a004218 PMID: 31604778; PMCID: PMC6824257.

27. Kurian AW, Ward KC, Howlander N, et al. Genetic testing and results in a population-based cohort of breast cancer patients and ovarian cancer patients. J Clin Oncol. 2019;37(15):1305-15.

28. Crawford B, Adams SB, Sittler T, et al. Multi-gene panel testing for hereditary cancer predisposition in unsolved high-risk breast and ovarian cancer patients. Breast Cancer Res Treat. 2017;163(2):383-90.

29. Domchek SM, Bradbury A, Garber JE, Offit K, Robson ME. Multiplex genetic testing for cancer susceptibility: out on the high wire without a net? J Clin Oncol. 2013;31(10):1267-70.

30. DaLuca H, Stuenkel A, Dolinsky JS, et al. Utilization of multigene panels in hereditary cancer predisposition testing: analysis of more than 2000 patients. Genet Med. 2014;16(11):830-7.

31. Yurgelun MB, Allen B, Kaldate RR, et al. Identification of a variety of mutations in cancer predisposition genes in patients with suspected lynch syndrome. Gastroenterology. 2015;149(3):604.

32. van Puijenbroek M, Nielsen M, Reinards TH, et al. The natural history of a combined defect in MSH6 and MUTYH in a HNPCC family. Familial Cancer. 2007;6(1):43-51.

33. Pearlman R, Frankel WL, Swanson B, et al. Prevalence and Spectrum of Germline Cancer susceptibility gene mutations among patients with earlyonset colorectal Cancer. JAMA Oncol. 2017;3(4):464-71.

34. Chatrath A, Przanowska R, Kiran S, et al. The pan-Cancer landscape of prognostic Germline variants in 10,582 patients. Genome Med. 2020; 12(1):15.

35. Benitez-Buelga $C$, Vaclová T, Ferreira $\mathrm{S}$, et al. Molecular insights into the OGG1 gene, a Cancer risk modifier in BRCA1 and BRCA2 mutations carriers. Oncotarget. 2016;7(18):25815-25.

36. Box N, Duffy D, Chen W, et al. MC1R genotype modifies risk of melanoma in families segregating CDKN2A mutations. Am J Hum Genet. 2001;69(4): $765-73$.

37. Okkels H, Sunde L, Lindorff-Larsen $\mathrm{K}$, et al. Polyposis and early Cancer in a patient with low penetrant mutations in MSH6 and APC: hereditary colorectal Cancer as a polygenic trait. Int J Color Dis. 2006;21(8):847-50.

38. Sarasin A, Kauffmann A. Overexpression of DNA repair genes is associated with metastasis: a new hypothesis. Mutat Res. 2008;659(1-2):49-55. 
39. Alvino E, Passarelli F, Cannavò E, Fortes C, Mastroeni S. High expression of the mismatch repair protein MSH6 is associated with poor patient survival in melanoma. Am J Clin Pathol. 2014;142(1):121-32.

40. Chen R, Shi L, Hakenberg J, Naughton B, Sklar P. Analysis of 589,306 genomes identifies individuals resilient to severe Mendelian childhood diseases. Nat Biotechnol. 2016;34(5):531-8.

41. Hall MJ, Forman AD, Pilarski R, Wiesner G, Giri VN. Gene panel testing for inherited Cancer risk. J Natl Compr Cancer Netw. 2014;12(9):1339-46.

\section{Publisher's Note}

Springer Nature remains neutral with regard to jurisdictional claims in published maps and institutional affiliations.

Ready to submit your research? Choose BMC and benefit from:

- fast, convenient online submission

- thorough peer review by experienced researchers in your field

- rapid publication on acceptance

- support for research data, including large and complex data types

- gold Open Access which fosters wider collaboration and increased citations

- maximum visibility for your research: over $100 \mathrm{M}$ website views per year

At $\mathrm{BMC}$, research is always in progress.

Learn more biomedcentral.com/submissions 